\title{
Superheavy Gravitinos and Ultra-High Energy Cosmic Rays
}

\author{
Krzysztof A. Meissner ${ }^{1}$ and Hermann Nicolai ${ }^{2}$ \\ ${ }^{1}$ Faculty of Physics, University of Warsaw \\ Pasteura 5, 02-093 Warsaw, Poland \\ ${ }^{2}$ Max-Planck-Institut für Gravitationsphysik (Albert-Einstein-Institut) \\ Mühlenberg 1, D-14476 Potsdam, Germany
}

Dedicated to the memory of Murray Gell-Mann

\begin{abstract}
We argue that the superheavy gravitinos that we had previously proposed as candidates for Dark Matter can offer a possible explanation for the ultra-high energy cosmic ray (UHECR) events observed at the Pierre Auger Observatory, via gravitino anti-gravitino annihilation in the 'skin' of neutron stars. The large mass and strong interactions of these particles, together with their stability against decays into standard matter are essential for the proposed explanation to work. In particular, it ensues that UHECR events can be understood to originate from neutron stars inside a GKZ horizon of $\sim 50 \mathrm{Mpc}$. The composition of neutron stars near their surface could play a crucial role in explaining the presence of heavy ions in these events. If confirmed, this new mechanism can be taken as evidence for the fundamental ansatz towards unification on which it is based.
\end{abstract}

To date there does not appear to exist a fully satisfactory explanation in terms of known physics for the ultra-high energy cosmic ray (UHECR) events observed over many years at the Pierre Auger Observatory [1-3], which reach maximum energies of $E \gtrsim 10^{19} \mathrm{eV}$ saturating the GKZ bound [4]. A particularly puzzling feature is the occurrence of not just protons, but heavier ions $(\mathrm{C}, \mathrm{N}, \mathrm{O}$, or heavier) which appear to dominate towards the very highest energies [1]. The absence of a compelling scenario may indicate the need for new physics beyond the Standard Model (SM). In this article we present a possible new explanation very different from previous proposals. This is based on our previous work [5, 6] where we have raised the possibility that dark matter (DM) could consist at least in part of an extremely dilute gas of very massive stable gravitinos, which are furthermore fractionally charged and strongly interacting. As we will argue here these can in principle furnish a fairly simple explanation for the most energetic cosmic ray energy events, both qualitatively and quantitatively.

Possible acceleration mechanisms relying on more conventional physics, such as Fermi acceleration of known particles by shock waves, have been amply discussed in the literature [3], but so far no clear picture has emerged. A more exotic possibility is to invoke the Penrose process of extracting energy from a rapidly rotating Kerr black hole 7]. Unfortunately, such an explanation runs into difficulties with Thorne's theorem [8], according to which rapidly spinning black holes obey $a / M \lesssim \beta_{\max }=0.998$ (where $a$ is the usual rotation parameter of the Kerr solution). It seems reasonable to assume that this bound also sets an upper limit for the velocity that a proton (or any other elementary particle) can acquire in such a process, but then the maximum attainable energy falls far short of the required $E \gtrsim 10^{19} \mathrm{eV}$ (corresponding to $1-\beta \sim 10^{-20}$ ). Another possible explanation could be via annihiliation of GUT-like objects, such as GUT mass magnetic monopoles. However, even assuming these do exist, it is not clear whether and how they could accumulate in sufficient amounts to explain the observed event rates. The same objection applies to other hypothetical GUT-like objects like leptoquarks or heavy gauge bosons, as these would most likely have decayed already long ago. In conclusion, there appears to be no compelling mechanism, neither from relativistic astrophysics nor from SM physics or widely discussed "Beyond the SM" scenarios, that could plausibly explain the acceleration of known (or suspected new) particles to the required energies, nor account for the observed abundance of heavy ions.

The new explanation proposed here is entirely different, being based on a more fundamental ansatz [5, 6]. That work was originally motivated by an attempt to explain the fermion content of the SM, with three generations of 16 quarks and leptons each, from the spin- $\frac{1}{2}$ fermion content of maximal $N=8$ supergravity, following a proposal orginally due to Gell-Mann [9, 10]. This proposal was further developed in [5, 12, 13] in order to fully account for the $\mathrm{SU}(3)_{c} \times \mathrm{SU}(2)_{w} \times \mathrm{U}(1)_{e m}$ assignments of the SM fermions, by exploiting properties of the maximal compact subgroup ('R symmetry') $K\left(E_{10}\right)$ of the conjectured maximal duality symmetry $E_{10}$. Just like $N=8$ supersymmetry, this group theoretical framework entails the existence of eight massive gravitinos in addition to the 48 fundamental spin- $\frac{1}{2}$ fermions. The present proposal is thus not simply based on ad hoc postulates, but part of a wider framework for unification with emergent space-time [11]; however, (Planck scale) supersymmetry is here not necessarily realized as a bona fide symmetry in the framework of space-time based quantum field theory. Although it so far relies solely on group theoretic considerations (whereas a proper dynamical description would require a much better understanding of the infinite-dimensional duality symmetries underlying it), one can nevertheless derive some interesting consequences from this kinematic framework even without detailed knowledge of the dynamics. 
Before continuing let us add a word of caution. Our main goal here is to point out a new possible mechanism for the generation of UHECRs, and not to present precise estimates for event rates (something other proposed schemes also cannot do). Although we do present some numerical estimates these are by no means meant to be definite predictions; rather the aim is to show that, with some reasonable assumptions, one can arrive at event rates that are not too far from the ones observed. There are many uncertainties, both known and unknown, about the actual physics, for instance concerning the composition and density profiles of neutron stars, unknown astrophysical input (such as the local distribution of DM), various unresolved issues of strong interaction dynamics, or extrapolations of known formulas (such as (17) below) far beyond their tested domains of validity. Any change in these numbers could significantly alter the final outcome. so the present exercise should be rather viewed as a "proof of principle". For this reason we will usually neglect factors of $\mathcal{O}(1)$ in the calculations below, as with current knowledge we anyhow cannot pretend to a higher level of precision.

The eight massive gravitinos are characterized by the following properties. From the group theoretic analysis given in [12, 13] it follows that they transform as

$$
\left(\mathbf{3}, \frac{1}{3}\right) \oplus\left(\overline{3},-\frac{1}{3}\right) \oplus\left(\mathbf{1}, \frac{2}{3}\right) \oplus\left(\mathbf{1},-\frac{2}{3}\right)
$$

under $\mathrm{SU}(3)_{c} \times \mathrm{U}(1)_{e m}$. These assignments follow from an $\mathrm{SU}(3) \times \mathrm{U}(1)$ decomposition of the $N=8$ supergravity gravitinos, except for the shift of the $\mathrm{U}(1)$ charges that was originally introduced in [9] to make the electric charge assignments of the spin- $\frac{1}{2}$ fermions agree with those of the quarks and leptons. As shown in [5, 12, 13], it is this latter shift which requires enlarging the $\mathrm{SU}(8) \mathrm{R}$ symmetry of $N=8$ supergravity to $K\left(E_{10}\right)$, and which takes the construction beyond $N=8$ supergravity. Hence, unlike DM candidates usually considered (such as axions or WIMPs), these particles do participate in strong and electromagnetic interactions, with coupling strengths of order $\mathcal{O}(1)$. All gravitinos are assumed to be supermassive with masses not too far from the (reduced) Planck mass $M_{\mathrm{Pl}} \sim 2 \cdot 10^{18} \mathrm{GeV} / \mathrm{c}^{2} \sim 4 \cdot 10^{-9} \mathrm{~kg}$ (in a supersymmetric context this would correspond to Planck scale breaking of supersymmetry). Finally, the charge assignments (1D) ensure that, despite their strong and electromagnetic interactions with ordinary matter, the superheavy gravitinos are stable because there are no fractionally charged final states in the SM into which they could decay in a way compatible with $\mathrm{SU}(3)_{c} \times \mathrm{U}(1)_{e m}$ and (1). Hence the only process that can lead to their disappearance is mutual annihilation, and this will be the crucial effect considered here.

However, there is an essential difference between the two kinds of gravitinos in (11). While the color singlet gravitinos would be pointlike objects (of size $\sim M_{\mathrm{Pl}}^{-1}$ ) the color triplet gravitinos are expected to form color singlet bound states with quarks, and thus very complicated dynamical objects that come with a gluonic cloud, which would effectively enhance their size from $M_{\mathrm{Pl}}^{-1}$ to $1 / \Lambda_{Q C D}$. We also note that because of their large mass these objects can be regarded as non-relativistic in all circumstances considered in this paper, whence intuition based on a parton picture does not apply, just as it does not apply to low energy protons - in particular, it makes no sense to attribute the mass of the bound state to its constituents in any particular way. There is (so far at least) basically nothing known about the dynamics of such putative superheavy strongly interacting objects, hence we will have to argue by analogy with known strong interaction physics, and to work with extrapolations of known and tested formulas to explore how the strongly interacting gravitinos can contribute to the annihiliation processes producing UHECR events.

Both the large gravitino mass and the amount of accumulated mass of these particles are necessary to understand the large energies and the rates of the observed UHECR events, as we shall now explain. In addition we need to make one important further assumption concerning the local distribution of DM in stellar systems. The average density of DM within a typical galaxy is commonly given as $\rho_{D M} \sim 0.3 \times 10^{6} \mathrm{GeV} \cdot \mathrm{m}^{-3}$ (corresponding to one proton per cubic centimeter) [14]. Extrapolating this number to Planck mass particles we would get $\sim 10^{-13}$ gravitinos per cubic meter, hence an extremely dilute gas of DM particles. Most of these will be color singlet gravitinos, whereas the strongly interacting non-singlets make up only a small fraction of these, see below. Now in [a] , we have already raised the possibility that DM, while more or less uniformly distributed in interstellar space, might be subject to larger local variations near stars. This could happen if the DM co-rotates with the stars around the center of the galaxy, but not relative to them, unlike the dust that gives rise to planets and ends up rotating around (and not being absorbed by) the star. Then, a typical star could eat up much of the surrounding DM in its vicinity over its lifetime, depleting a ball of diameter of a few lightyears around it. Taking half of the average distance between two stars as a rough guide this would yield a volume of (two lightyears) ${ }^{3} \sim 10^{49} \mathrm{~m}^{3}$ which in turn would imply a total amount of DM within the star of $\lesssim 10^{27} \mathrm{~kg}$, corresponding to a fraction of $\lesssim 10^{-3}$ of its total mass for a pre-supernova star. Although seemingly non-negligible it corresponds to only one gravitino per $10^{22}$ protons or helium nuclei. This number is far too small to affect standard stellar processes in any significant way, especially since our gravitinos cannot decay into SM particles. As participants in strong and electromagnetic interactions, they would nevertheless be in thermal equilibrium, with a very small velocity dispersion of $(\Delta v)^{2} \sim k T / M_{\mathrm{Pl}}$. In contradistinction to luminous matter (for which $\Delta v$ is very large), they would thus continue to slowly migrate towards the center of the star, especially if the latter develops a core of heavier nuclei, thereby avoiding the usual problem of 'missing the center' [15]. 
At this stage the gravitino density is still so small that annihilation processes can be neglected. Furthermore, because the color singlet gravitinos in (1) interact only electromagnetically their annihilation cross section is proportional to the inverse mass squared $\left(\sigma v \sim\left(\pi \alpha^{2} \hbar^{2} /\left(4 M^{2} c\right)\right.\right.$ for small initial velocities), hence of no significance to the effects considered here. The situation is expected to be entirely different for the strongly interacting gravitinos (or rather the associated color singlet bound states) corresponding to the two color triplets in (11), so let us explain in more qualitative terms why this is so. Like standard QCD bound states these composite particles would come with a strongly interacting cloud of gluons, and it is this fact that enhances the cross section. This can be seen by inspection of the annihilation cross section for heavy strongly interacting particles. From unitarity arguments one can derive the following formula for the inelastic cross section $\sigma_{\text {inel }}$ [16], which for particle anti-particle collisions at sufficiently small kinetic energies is the same as the annihilation cross section,

$$
\sigma_{\text {inel }}=\frac{\pi}{k^{2}\left(2 s_{1}+1\right)\left(2 s_{2}+1\right)} \sum_{j} \sum_{l, s, n}(2 j+1) X_{j \ell s n}
$$

where [16]

$$
X_{j \ell s n}=1-|\langle\ell s n|\mathbf{S}| \ell s n\rangle|^{2}-\sum_{\ell^{\prime} \neq \ell, s^{\prime} \neq s}\left|\left\langle\ell^{\prime} s^{\prime} n|(\mathbf{1}-\mathbf{S})| \ell s n\right\rangle\right|^{2}
$$

where $\mathbf{S}$ denotes the $S$-matrix $(j, \ell, s$ are the usual angular momentum labels, while $n$ stands for any other quantum numbers). The crucial point is now that for pointlike (e.g. electromagnetic) interactions, only the lowest angular momenta are relevant, so that with $\mathbf{k}^{2}=M^{2} v^{2} / 4$ we get the expected result $\sigma_{\text {inel }} v \sim 1 /\left(M^{2} v\right)$. By contrast, for strongly interacting particles many more angular momentum states may contribute to the sum in (2): the known cross section for proton anti-proton collisions suggests that angular momenta get excited up to some maximum $j_{\max } \sim M / \Lambda_{\mathrm{QCD}}$. Then the sum over angular momenta can compensate the smallness of the factor $1 / M^{2}$ to replace it by $1 / \Lambda_{\mathrm{QCD}}^{2}$, whence we obtain

$$
\sigma_{\text {inel }} v \sim \frac{1}{\Lambda_{Q C D}^{2}}
$$

One can then fit the known proton-antiproton annihilation cross section to get the approximate formula [17]

$$
\langle\sigma \beta\rangle \sim \Lambda_{Q C D}^{-2}\left[3.84-0.51 \ln \left(\frac{\sqrt{s}}{\Lambda_{Q C D}}\right)+0.084\left(\ln \left(\frac{\sqrt{s}}{\Lambda_{Q C D}}\right)\right)^{2}\right]
$$

with $\Lambda_{Q C D}=0.2 \mathrm{GeV}$. For $\sqrt{s}=\Lambda_{Q C D}$ we obtain $\langle\sigma \beta\rangle \sim 38 \mathrm{mb}$. This formula is non-perturbative in the sense that it does not rely on a perturbative calculation, but rather on imposing the Froissart bound and fitting the relevant parameters to the data over the whole range of available energies (notice that the dependence on $s$ is not very pronounced). Putting $\sqrt{s}=2 \gamma m_{p}$ and $\gamma \sim 1$ we find $\langle\sigma \beta\rangle \sim 32 \mathrm{mb}\left(1 \mathrm{mb} \sim 10^{-31} \mathrm{~m}^{2} \sim 2.5 \mathrm{GeV}^{-2}\right)$. Again reasoning by analogy, we will use this value also for gravitino antigravitino annihilation to get an order of magnitude estimate.

To estimate the present density $\rho_{0}$ of strongly interacting (color triplet) gravitinos, we observe that with a gravitino mass close to $M_{\mathrm{Pl}}$, the usual requirement of thermal equilibrium reads

$$
\Gamma=\rho\langle\sigma v\rangle>H=\frac{\pi\left(k_{B} T\right)^{2}}{3 \sqrt{5} \hbar c^{2} M_{\mathrm{Pl}}}
$$

Adopting from now on the usual unit conventions $\hbar=c=k_{B}=1$ (hence $2 \cdot 10^{-7} \mathrm{eV} \cdot \mathrm{m}=1$ ), and using the values for $\sigma$ given above this translates into an equation for the relic abundance $\rho_{T}$

$$
(32 \mathrm{mb}) \rho_{T} \equiv(32 \mathrm{mb}) g\left(\frac{m T}{2 \pi}\right)^{3 / 2} \mathrm{e}^{-m / T}=\frac{T^{2}}{2 M_{\mathrm{Pl}}}
$$

( $g=4$ for a massive gravitino), or

$$
\frac{m}{T} \sim 90 \Rightarrow \rho_{T} \sim 3 \cdot 10^{59} \mathrm{~m}^{-3}
$$

The temperature $T \sim 2 \cdot 10^{16} \mathrm{GeV}$ corresponds to cosmic time $t_{T}=M_{\mathrm{Pl}} / T^{2} \sim 3 \cdot 10^{-39} \mathrm{~s}$. The present density $\rho_{0}$ is obtained from $\rho_{T}$ by the well known formula

$$
\rho_{0}=\rho_{T}\left(\frac{a_{T}}{a_{0}}\right)^{3}
$$


(since superheavy gravitinos are non-relativistic). Taking the end of the radiation dominated era as $10^{12} \mathrm{~s}$ we get

$$
\frac{a_{T}}{a_{0}}=\left(\frac{3 \cdot 10^{-39}}{10^{12}}\right)^{1 / 2}\left(\frac{10^{12}}{3 \cdot 10^{17}}\right)^{2 / 3} \sim 10^{-29}
$$

where the two factors correspond to the radiation dominated and matter dominated eras, respectively. Thus

$$
\rho_{0} \sim 5 \cdot 10^{-28} \mathrm{~m}^{-3} \quad\left(\sim 10^{-9} \mathrm{GeV} \cdot \mathrm{m}^{-3}\right)
$$

Assuming now (as before) that the star 'swallows' all gravitinos within a radius of two lightyears we get for the total number of color triplet gravitinos inside the star

$$
N_{g} \sim 2 \cdot 10^{22}
$$

As we argued above, the gravitinos inside the star are in thermal, but not mechanical equilibrium, so for a presupernova star we expect them to cluster more towards the iron core (where no nuclear reactions take place anymore). As already pointed out, the number (12) is too small to produce any significant effects in the star - even in the iron core the lifetime of gravitinos via annihilation still exceeds the lifetime of the Universe, see below.

The situation changes dramatically if the star collapses to a neutron star. In that case, as explained above, most of the gravitinos will be contained in its iron core even prior to the supernova collapse, and the gravitinos will collapse with the core due to the sudden increase of gravititional pull towards the center. As a consequence, they will get squeezed into a ball of radius $\mathcal{O}(10 \mathrm{~km})$ [18], and their density increases to

$$
\rho_{N S} \sim 5 \cdot 10^{9} \mathrm{~m}^{-3}
$$

This 'compactification' is absolutely crucial since the gravitinos need to be packed sufficiently closely to enable them to annihilate in any appreciable rate. The inverse lifetime of the gravitino as a function of the neutron star time from its birth is

$$
\Gamma_{N S}(t)=\rho_{N S} \exp \left(-\int_{0}^{t} \Gamma_{N S}\left(t^{\prime}\right) \mathrm{d} t^{\prime}\right)\langle\sigma v\rangle
$$

which gives

$$
\Gamma_{N S}(t)=\frac{\Gamma_{N S}(0)}{1+\Gamma_{N S}(0) t}
$$

with the initial value (and $\langle\sigma \beta\rangle \sim 32 \mathrm{mb}$ )

$$
\Gamma_{N S}(0) \sim\left(5 \cdot 10^{9}\right) \cdot\left(32 \cdot 10^{-31}\right) \cdot\left(3 \cdot 10^{8}\right) \mathrm{s}^{-1} \sim 5 \cdot 10^{-12} \mathrm{~s}^{-1}
$$

Therefore the actual annihilation rate depends on the age of the neutron star. We also see that before the collapse for a pre-supernova star with an iron core of $\mathcal{O}(1000 \mathrm{~km})$ diameter the rate would be lower by a factor of $\sim 10^{-12}$.

Having derived the approximate annihilation rate inside the neutron stars we can now estimate the number of UHECR particles coming from the annihilation. Because the superheavy gravitinos interact strongly, each single annihilation will result in a violent burst of Planck scale energy, producing a multitude of (mostly hadronic) particles. We can roughly estimate their multiplicity by extrapolating to Planckian energies the formula [19]

$$
\text { multiplicity } \sim 0.27 \alpha_{s}(\Lambda) \exp \left(\frac{2.26}{\sqrt{\alpha_{s}(\Lambda)}}\right)
$$

whose validity has been confirmed for proton-proton collisions from the lowest energies to the highest energies attainable by LHC; this must suffice for the present purposes as no other formula seems to be available for the much larger energies considered here. The strong coupling $\alpha_{s}$ is to be evaluated at $\Lambda \sim 0.35 \sqrt{s}$. Plugging in $\sqrt{s} \sim M_{\mathrm{Pl}}$ gives $\mathcal{O}\left(10^{6}\right)$ particles per annihilation (whereas for the cross section in (5) the relevant quantity is the relative velocity $\gamma$ which in our case is very close to one). The total energy $\sim M_{\mathrm{Pl}}$ will be distributed over all these particles, with an average energy of $10^{13} \mathrm{GeV} \sim 10^{22} \mathrm{eV}$ per particle. This happens to be of the same order of magnitude as the maximum energy observed in high energy cosmic rays! Nevertheless, because of their strong interactions and the large density inside the neutron star the annihilation products cannot escape because they will either lose too much energy or be stopped altogether on their way out from the core of the neutron star. For this reason, we expect the main 
contribution to UHECR particles to come from the outermost shell of the neutron star of width $d \lesssim \mathcal{O}(100 \mathrm{~m})$ i.e. $\sim 3 \%$ of the volume. There the density drops down by a factor $10^{-5}$ relative to the core density [18], and is given by $\sim 10^{13} \mathrm{~kg} \mathrm{~m}^{-3}$ such that $\rho(R-d)=10^{40} \mathrm{~m}^{-3}$. (The width $d$ is here determined by the requirement that the number of collisions times the loss of energy per collision, here assumed to be $\sim \mathcal{O}(1 \mathrm{GeV})$, should be much lower than the total energy of the proton of $10^{12} \mathrm{GeV}$, which gives $\rho(R-d) \sigma d<10^{12}$, where $\left.\sigma \sim 10^{-30} \mathrm{~m}^{2}\right)$.

Importantly, this outer shell is thought to be rich in heavier nuclei, including iron nuclei [18], so the high energy particles that can escape may 'sweep up' hadrons as well as heavier ions before exiting the neutron star. Whether and how such a 'sweep-up' could work in detail remains to be explored, as this would require a much better understanding of difficult issues in neutron star and strong interaction physics. Moreover, no data that could be relevant to such processes appear to be available in the published literature (for instance, the ALICE experiment at CERN does not provide any information on heavy fragments with large longitudinal momentum resulting from the proton-lead collisions). If indeed a sufficient abundance of such highly energetic fragments could be generated and get out of the neutron star, the resulting heavier ions will subsequently decay to a variety of stable isotopes, and thus end up as ultra-high energy stable ions of the type observed by [1].

Despite the fall-off of the density profile towards the outer regions of the neutron star the density of gravitinos near the skin may actually be enhanced by a 'centrifuge effect' for rapidly spinning neutron stars. These two effects would have to compete with each other in determining the gravitino density, but due to the lack of sufficiently detailed information on the physics of neutron stars not much more can be said at this point. For this reason we shall simply take the value (13) to hold also near the skin of the neutron star, and assume that $3 \%$ of the neutron star volume is effectively available for this process. A young neutron star would thus continuously 'spray' high energy protons or heavy ions at a rate

$$
\sim 6 \cdot 0.03 \cdot\left(2 \cdot 10^{22}\right) \cdot\left(5 \cdot 10^{-12}\right) \cdot 10^{6} \mathrm{~s}^{-1} \sim 2 \cdot 10^{16} \mathrm{~s}^{-1}
$$

from its surface into outer space (the factor of 6 comes from the number of the strongly interacting gravitino species). To calculate how many of these will eventually reach Earth, we recall that, with an estimated average number of neutron stars per galaxy of $\sim 10^{8}$ and $\sim 10^{7}$ galaxies within a GKZ horizon of $50 \mathrm{Mpc}[$ ] , we have a total number of $10^{15}$ UHECR emitters. Denoting the density of neutron stars in the universe by $\rho_{N}(\mathbf{x})$ (where $\mathbf{x}=0$ corresponds to the position of Earth), the total rate arriving at Earth is thus

$$
N_{E} \sim\left(2 \cdot 10^{16} s^{-1}\right) \times \int \frac{\rho_{N}(\mathbf{x}) d^{3} x}{4 \pi|\mathbf{x}|^{2}}
$$

For a rough estimate of the total flux we neglect density variations, taking $\rho_{N}=$ const, in which case the integral is easily evaluated to be

$$
N_{E} \sim \rho_{N} R_{\max } \times 2 \cdot 10^{16} \mathrm{~s}^{-1}
$$

Putting $R_{\max } \sim 50 \mathrm{Mpc}$ as a cutoff we arrive at the flux of UHECR arriving on Earth as

$$
N_{E} \sim \frac{10^{15} \cdot 2 \cdot 10^{16}}{4\left(10^{24}\right)^{2}} \mathrm{~m}^{-2} \mathrm{~s}^{-1} \sim 5 \cdot 10^{-18} \mathrm{~m}^{-2} \mathrm{~s}^{-1}
$$

which is not too far off the observed rate of one UHECR event per month and per $3000 \mathrm{~km}^{2}$ [1]. To be sure, the UHECR emitters are not evenly distributed throughout the universe, and we therefore expect an increased number of events to originate from superclusters of galaxies rich in neutron stars (the supergalactic plane, in particular, as also suggested by the data [1]). In particular the integral in (19) may receive its dominant contribution from a disk rather than the full ball. We also note that with a maximum available energy of $\mathcal{O}\left(10^{22} \mathrm{eV}\right)$ our proposal can also explain the existence of (very rare) UHECR events exceeding the GKZ bound, if these originate from neutron stars within the Milky Way or nearby galaxies.

We thus arrive at an explanation which agrees qualitatively with observations, and at an estimated event rate that albeit subject to some crucial assumptions, is not too far from the one observed. Evidently, there remain many uncertainties in our calculation, quite apart from questions concerning the viability of the unification scenario proposed in [5]. Nevertheless, we find it remarkable that the present proposal could tie in with the scheme proposed in [5] to explain the fermion content of the SM, with three generations of quarks and leptons.

Acknowledgments: We are most grateful to Masaru Shibata and Kacper Zalewski for enlightening discussions. K.A. Meissner thanks AEI for hospitality and support; he was partially supported by the Polish National Science Center grant DEC-2017/25/B/ST2/00165. The work of H. Nicolai has received funding from the European Research Council (ERC) under the European Union's Horizon 2020 research and innovation programme (grant agreement No $740209)$. 
[1] The Pierre Auger Collaboration, arXiv:1107.4804;

S. Petrera, arXiv:1903.00529, and references therein.

[2] P. Bhattacharjee and G. Sigl, Phys.Rept. 327 (2000) 109

[3] K. Kotera and A.V. Olinto, Ann. Rev. Astron. Astrophys. 49 (2010), arXiv:1101.4256[astro-ph.HE]

[4] K. Greisen, Phys. Rev. Lett. 16 (1966) 748, 1966;

G. T. Zatsepin and V. A. Kuzmin, Sov. Phys. JETP Lett. 4 (1966) 78.

[5] K.A. Meissner and H. Nicolai, Phys. Rev. Lett. 121 (2018) 091601

[6] K.A. Meissner and H. Nicolai, Phys. Rev. D100 (2019) 035001

[7] R. Penrose and R.M. Floyd, Nature Physical Science 229 (1972) 177

[8] K. Thorne, Astrophys. Journal 191 (1974) 507

[9] M. Gell-Mann, in Proceedings of the 1983 Shelter Island Conference on Quantum Field Theory and the Fundamental Problems of Physics, eds. R. Jackiw, N.N. Khuri, S. Weinberg and E. Witten, Dover Publications, Mineola, New York (1985)

[10] H. Nicolai and N.P. Warner, Nucl. Phys. B259 (1985) 412

[11] T. Damour, M. Henneaux and H. Nicolai, Phys. Rev. Lett. 89 (2002) 221601

[12] K.A. Meissner and H. Nicolai, Phys. Rev. D91 (2015) 065029

[13] A. Kleinschmidt and H. Nicolai, Phys. Lett. B747 (2015) 251

[14] M. Weber and W. de Boer, Astron.Astrophys. 509 (2010) A25

[15] J.N. Bahcall, R.A. Wolf, Astrophys. J. 209 (1976) 214

[16] Lam Hui, Phys.Rev.Lett. 86 (2001) 3467

[17] T. Wibig and D. Sobczyńska, J.Phys. G24 (1998) 2037

[18] S.L. Shapiro and S.A. Teukolsky, Black Holes, White Dwarfs, and Neutron Stars, Wiley, New York, 1983.

[19] J.F. Grosse-Oetringhaus, K. Reygers, J.Phys G37 (2010) 083001 